\title{
Acoustics of a Stratified Poroelastic Composite
}

\author{
R. P. Gilbert and A. Panchenko
}

\begin{abstract}
In this paper we discuss the acoustic boundary layer problem for a poroelastic seabed abutting onto a liquid half space. The problem is addressed using the method of homogenization where the microscopic equations are modelled after Burridge and Keller [5], Levy [10], and Panasenko [13]. A difference in our approach is that we do not consider the viscosity coefficients to be dependent on the pore size. To achieve continuity of displacement and stress at the interface to an arbitrary asymptotic order, we introduce correctors of two different types on each side. Then correctors of different types are matched across the interface.
\end{abstract}

Keywords: Homogenization, asymptotic expansions, boundary layers, composite materials AMS subject classiflcation: 74 Q 10, 35 Q 35

\section{Introduction and Remarks}

In this paper we discuss the vibrational motion of a porous medium whose pore space is saturated with fluid. The porous medium we propose to study is formed by a periodic arrangement of the pores into cells. The vibrational motion is assumed to be stimulated acoustically by a signal whose wave length is $\lambda$. For an averaging procedure to work, we need the wavelength to be large compared to a typical cell size $\ell$. Assuming in addition that $\lambda$ is comparable to the characteristic macroscopic size $L$ of the problem and the fluid phase is incompressible, one can classify different homogenized models, as was done in [2] heuristically, and justified rigorously in [7]. In these works, four different types of possible macroscopic behavior are listed:

- Model I: The acoustics of a fluid in a rigid porous matrix regime. This case was considered previously by Gilbert and Panasenko [6].

- Model II: Diphasic macroscopic behavior of the fluid and solid matrix. This case is considered using the methods of two-scale convergence in [7].

- Model III: Monophasic elastic macroscopic behavior. This case is also discussed in [7].

- Model IV: Monophasic viscoelastic macroscopic behavior.

Model II, the diphasic case corresponds to the Biot model $[3,4]$.

In this paper, we allow the fluid to be compressible and do not assume that $\lambda$ is comparable to $L$. Thus the model developed in the paper will also work for $\lambda$ large

Both authors: University of Delaware, Dept. Math. Sci.; Newark, DE 19716, U.S.A. Authors were partially supported by the NSF grant BES-9402539.

ISSN 0232-2064 / \$ 2.50 @ Heldermann Verlag Berlin 
compared to $\ell$ but small compared to $L$. The homogenized model obtained is close to the "slightly compressible" variant of model III as developed in [7]. The microscopic equations we use are similar to the ones used in papers by Levy [10] and Burridge and Keller [5]. The only, but essential difference is that we do not assume the viscosity coefficients to be comparable to the pore size. We adopt a point of view according to which viscosity characterizes properties of the fluid regardless of the pore geometry. The immediate conclusion then is that the classical Biot model is an approximation valid for pore size lying within a certain range. The latter is determined by the viscosity of the fluid and by the geometric characteristics of the medium. Passing to the limit of "infinitely small" pore size, one should expect to obtain a set of equations different from $[3,5,10]$.

Denote the ratio between $\ell$ and $L$ is given by $\varepsilon, \varepsilon L=\ell$. The geometrical structure inside the unit cell $Q=(0,1)^{n}$ has a solid part $Q^{s}$ which is a closed subset of $\bar{Q}$, and a fluid part $Q^{f}=Q \backslash Q^{s}$. Now we assume $Q^{s}$ is periodically repeated over $\mathbf{R}^{n}$ and set $Q_{k}^{s}=Q^{s}+k$ for $k \in \mathbf{Z}^{n}$. Obviously, the obtained closed set $X^{s}=\bigcup_{k \in \mathbf{Z}^{n}} Q_{k}^{s}$ is a closed subset of $\mathbf{R}^{n}$ and $X^{f}=\mathbf{R}^{n} \backslash X^{s}$ in an open set in $\mathbf{R}^{n}$. We make the following assumptions on $Q^{f}$ and $X^{f}$ :

(i) $Q^{f}$ is an open connected set of strictly positive measure with a smooth boundary, and $Q^{s}$ has strictly positive measure in $\bar{Q}$ as well.

(ii) $X^{f}$ and the interior of $X^{s}$ are open sets with boundary of class $C^{\infty}$, which are locally situated on one side of their boundary. Moreover, $X^{f}$ is connected.

Now we see that $X=(0, L)^{n}$ is covered with a regular mesh of size $\varepsilon$, each cell being a cube $Q_{i}^{\epsilon}$, with $1 \leq i \leq N(\varepsilon)=|X|(\varepsilon)^{-n}[1+0(1)]$. Each cube $Q_{i}^{\epsilon}$ is homeomorphic to $Q$, by a linear homeomorphism $\Pi_{i}^{\varepsilon}$, being composed of translation and an homothety of ratio $\frac{1}{\epsilon}$. We define

$$
Q_{S_{i}}^{\varepsilon}=\left(\Pi_{i}^{\varepsilon}\right)^{-1}\left(Q^{s}\right) \quad \text { and } \quad Q_{f_{i}}^{\varepsilon}=\left(\Pi_{i}^{\varepsilon}\right)^{-1}\left(Q^{f}\right)
$$

For sufficiently small $\varepsilon>0$ we consider the sets

$$
T_{\varepsilon}=\left\{k \in \mathbf{Z}^{n} \mid Q_{S_{k}}^{e} \subset X\right\} \quad \text { and } \quad K_{\varepsilon}=\left\{k \in \mathbf{Z}^{n} \mid Q_{S_{k}}^{e} \operatorname{cap} \partial X \neq \emptyset\right\}
$$

and define

$$
X_{s}^{\varepsilon}=\bigcup_{k \in T_{c}} Q_{S_{k}}^{e}, \quad S^{\varepsilon}=\partial X_{s}^{\varepsilon}, \quad X_{f}^{\varepsilon}=X \backslash X_{s}^{e} .
$$

Obviously, $\partial X_{f}^{\varepsilon}=\partial X \cup S^{\varepsilon}$. The domains $X_{g}^{\varepsilon}$ and $X_{f}^{\varepsilon}$ represent, respectively, the solid and fluid parts of a porous medium $X$. For simplicity we suppose $\frac{L}{\varepsilon} \in \mathbf{N}$. Then $K_{\varepsilon}=\emptyset$.

We construct a full asymptotic expansion for the system of two composites of the above type, separated by a plane interface. The first part of the construction deals with inner expansions. The development here is based on the general method proposed by Panasenko [13]. Then we proceed to take care of the boundary layers at the interface.

The major part of this work is devoted to the investigation of interface effects between two different composites. We consider the simplest model situation of a single plane interface. The goal is again to produce a full asymptotic expansion accurate 
to an arbitrary power of the parameter, which normally is a difficult task. A simple computation shows that the usual inner expansions can not be matched at the first level, even after the introduction of boundary layer correctors of a type typically employed in the literature. In classical works on homogenization such as [15] this problem is discussed, but the only rigorous work known to the authors is the recent paper by Avellaneda, Berlaynd, and Clouet [1], where the problem of interface matching is solved under the assumption that wave propagation can be described by wave equations at the microscopic level. The authors use the Floquet theory and operator perturbation theory to obtain explicit asymptotics of the Dirichlet-to-Neumann map at the interface.

The main idea that makes matching possible is the introduction of two different correctors on each side. The first corrector is a usual one, so we call it "old". The second, "new" corrector combines the fast variable part of the expansion on this side and the slow variable part of the inner expansion from the opposite side. The matching is done as follows: the old corrector from one side is matched with the new corrector from the opposite side. This is the crucial part of the method. On the output, this "microscopic" matching produces two families of constant matrices used to obtain macroscopic matching conditions. The constants can be determined explicitly from the formula contained in Theorem 4.1, and also from the examination of the proof of Theorem 5.3. As an example, we calculate homogenized transmission conditions for the main term of the expansion. The result shows that the homogenized transmission conditions can be written in terms of homogenized normal stress only. The conditions for subsequent terms contain non-trivial corrections to the homogenized stress, which shows significance of edge effects. The details can be found in Section 4.

In order to control decay of boundary layer correctors, one needs to estimate solutions of the cell problem in unbounded domains. In the present case the cell problem is a system of stationary viscoelasticity. The problem to solve is twofold. First, we need to obtain sufficient conditions for the existence of solutions with derivatives which do not grow too fast. Then, imposing some extra conditions, we show that derivatives will decay exponentially, and the solution itself will stabilize to a constant vector with exponential speed. This is the most difficult and technical part of the paper. It is based on special a priori estimates of Saint-Venant type. Estimates of this kind for the elasticity system were obtained by Oleinik and Yosifian [11]. In the paper, we obtain a generalization of these estimates, since we need to deal with complex-valued coefficients. Moreover, the estimates in [11] are written in a half-space. In order to control the decay of solutions of the transmission problem, we need to estimate the decay of solutions on both sides of the interface, rather than on just one. We point out, however, that most of the technical arguments from [11] still works.

The paper is organized as follows. In Section 2 we discuss the system of equations to be homogenized, and a certain transformation that leads to the elimination of the pressure from the system. In Section 3, the inner expansion is obtained. Section 4 contains the details of the interface matching procedure. Finally, in the technical Section 5 we give proofs of the estimates needed in Section 4. 


\section{System of equations for acoustics in a periodic porous medium}

Consider an infinite $\varepsilon$-periodic medium composed of an elastic solid and a compressible viscous fluid. Let $X^{s}$ and $X^{f}$ denote the domains occupied by the solid and fluid, respectively. Their common boundary $S$ is assumed to be a smooth manifold of codimension one. The displacement vector $u$ satisfies the system of equations (written componentwise):

$$
\begin{aligned}
\rho^{s} \frac{\partial^{2} u_{i}}{\partial t^{2}}=\frac{\partial \sigma_{i j}^{s}}{\partial x_{j}}+f_{i} & \text { in } X^{s} \\
\rho^{f} \frac{\partial^{2} u_{i}}{\partial t^{2}}=\frac{\partial \sigma_{i j}^{f}}{\partial x_{j}}+f_{i} & \text { in } X^{f} .
\end{aligned}
$$

Moreover, on the interface $S$, the transmission conditions

$$
\left.\begin{array}{r}
{[u]=0} \\
{\left[\sigma_{i j} \nu_{j}\right]=0}
\end{array}\right\}
$$

hold, where $\nu_{j}$ denote components of unit normal to $S$ pointing inside of $X^{s}$. In the solid part, components of the stress tensor $\sigma^{s}$ satisfy the Hooke law

$$
\sigma_{i j}^{s}=a_{i j k l}^{s} e_{k l}(u), \quad e_{k l}(u)=\frac{1}{2}\left(\frac{\partial u_{k}}{\partial x_{l}}+\frac{\partial u_{l}}{\partial x_{k}}\right)
$$

with coefficients $a_{i j k l}^{s} \in C^{\infty}\left(X^{s}\right)$ satisfying conditions of symmetry and positivity:

$$
\begin{gathered}
a_{i j k l}^{s}=a_{j i k l}^{s}=a_{j i k}=a_{k l i j}^{s} \\
k_{1} e_{i j}(u) e_{i j}(u) \geq a_{i j k l}^{s} e_{i j}(u) e_{k l}(u) \geq k_{2} e_{i j}(u) e_{i j}(u) .
\end{gathered}
$$

In the fluid part $X^{f}$, the stress tensor satisfies the Navier-Stokes law

$$
\sigma_{i j}^{f}=-\delta_{i j} P+\left(\lambda \delta_{i j} \delta_{k l}+2 \mu \delta_{i k} \delta_{j l}\right) e_{k l}\left(\frac{\partial u}{\partial t}\right)
$$

where the viscosity coefficients $\mu$ and $\lambda$ satisfy

$$
\mu>0, \quad \frac{\lambda}{\mu}>-\frac{2}{3} k_{2}, \quad 0<k_{2}<1
$$

If the displacement is small, we can linearize the equation near the reference state characterized by the known reference densities $\rho^{s}$ and $\rho^{f}$. The linearized equation of state relates pressure $P$ to the perturbation of density $\rho$ :

$$
P=c^{2} \rho
$$


where $c$ is the speed of sound. Moreover, the linearized conservation of mass equation gives a relation between $\rho$ and the velocity $\frac{\partial u}{\partial t}$ :

$$
\frac{\partial \rho}{\partial t}+\rho^{f} \operatorname{div} \frac{\partial u}{\partial t}=0
$$

Integrating, we obtain $\rho+\rho^{f} \operatorname{div} u=0$. Combining this and (2.8) we have $P=$ $-c^{2} \rho^{f} \operatorname{div} u$, so that the pressure can be eliminated from (2.6):

$$
\sigma_{i j}^{f}=a_{i j k l}^{f} e_{k l}(u)
$$

where

$$
a_{i j k l}^{f}=-c^{2} \rho^{f} \delta_{i j} \delta_{k l}+\left(\lambda \delta_{i j} \delta_{k l}+2 \mu \delta_{i k} \delta_{j l}\right) \frac{\partial}{\partial t}
$$

Denote by $a_{i j k l}$ the components of thew symmetric fourth-order tensor equal to $a_{i j k l}^{s}$ in $X^{s}$ and to $a_{i j k l}^{f}$ in $X^{f}$, and denote by $\sigma$ the corresponding "unified" symmetric stress tensor. Since $e(u)$ is also symmetric, we can write

$$
\sigma_{i j}=a_{i j k l} \frac{\partial u_{l}}{\partial x_{k}}
$$

Then

$$
\frac{\partial \sigma_{i j}}{\partial x_{j}}=\frac{\partial}{\partial x_{j}}\left(M_{k j} \frac{\partial u}{\partial x_{k}}\right)
$$

where $M_{k j}$ are $n \times n$-matrix operators with components $a_{i j k l}$. Using matrices $M_{k j}$ we can replace (1.1) and (2.2) by a single system:

$$
\rho^{\circ} \frac{\partial^{2} u}{\partial t^{2}}-\frac{\partial}{\partial x_{k}}\left(M_{k j} \frac{\partial u}{\partial x_{j}}\right)=f
$$

where $\rho^{0}$ equals to $\rho^{\mathbf{s}}$ in $X^{s}$, and to $\rho^{f}$ in $X^{f}$. The components $a_{i j k l}$ of the matrices $M_{k j}$ satisfy (2.5) and (2.10) in $X^{s}$ and $X^{f}$, respectively. On the interface $S$, the transmission conditions (2.3) hold.

\section{Inner expansion and homogenized system}

The purpose of this section is to obtain a complete inner asymptotic expansion for a solution of system (2.11). The word "inner" means that at the moment we prescribe no macroscopic boundary conditions and treat the medium as infinite in all directions. The terms of the expansion will satisfy a chain of problems with coefficients independent of the parameter. The first system in this chain provides the macroscopic homogenized equations.

Consider system (2.11) together with the interface conditions (2.3). Assuming that $u$ is a time-harmonic vector with angular frequency $\omega$, and slightly abusing notation, we replace $u(x, t)$ by $u(x, \omega) c^{i \omega t}$. Then the amplitude $u(x, \omega)$ satisfies

$$
-\omega^{2} \rho u-\frac{\partial}{\partial x_{j}}\left(A_{k j} \frac{\partial u}{\partial x_{k}}\right)=f
$$


where $u(x)$ is an $n$-component vector function of $x, A_{k j}\left(\frac{x}{\ell}, \omega\right)$ are periodic $n \times n$ matrices with components $A_{k j}^{(i)}$ given by

in $X^{s}$, and by

$$
A_{k j}^{(i l)}=a_{i j k l}^{s}
$$

$$
A_{k j}^{(i l)}=\left(-c^{2} \rho^{f}+i \omega \lambda\right) \delta_{i j} \delta_{k l}+2 i \omega \mu \delta_{i k} \delta_{j l}
$$

in $X^{f}$. By assumption, $A_{k j}$ are smooth everywhere in $\mathbf{R}^{n}$ except a smooth manifold $S$ of codimension one. On $S$, the transmission conditions

$$
\left.\begin{array}{rl}
{[u]} & =0 \\
{\left[A_{k j}^{i l} \frac{\partial u_{l}}{\partial x_{k}} \nu_{j}\right]} & =0 \quad(i=1,2, \ldots, n)
\end{array}\right\}
$$

are satisfied, where $\nu_{j}$ are components of the unit normal to $S$. We look for an asymptotic in $\varepsilon$ solution of the form

$$
u \sim \sum_{p, l=0}^{\infty} \varepsilon^{p+l} \sum_{|i|=l} N^{p, i}(\xi) D^{i} v(x)
$$

By the chain rule, the differential operator

$$
L_{x}=\frac{\partial}{\partial x_{j}}\left(A_{k j} \frac{\partial}{\partial x_{k}}\right)
$$

corresponds to

$$
\varepsilon^{-2} L_{\xi}+\varepsilon^{-1}\left[A_{k j}\left(\frac{\partial^{2}}{\partial x_{j} \partial \xi_{k}}+\frac{\partial^{2}}{\partial x_{k} \partial \xi_{j}}\right)+\frac{\partial A_{k j}}{\partial \xi_{j}} \frac{\partial}{\partial x_{k}}\right]+A_{k j} \frac{\partial^{2}}{\partial x_{j} \partial x_{k}}
$$

Substituting (3.4) into system (3.1), and taking (3.5) into account, we obtain

$$
\begin{aligned}
f \sim & \sum_{l, p=0} \varepsilon^{l+p} \sum_{|i|=l}-\omega^{2} \rho N^{p, i} D^{i} v+\sum_{l, p=0} \varepsilon^{l+p-2} \sum_{|i|=l} L_{\xi} N^{p, i} D^{i} v \\
& +\sum_{l, p=0} \varepsilon^{l+p-1} \sum_{|i|=l} A_{k j} \frac{\partial N^{p, i}}{\partial \xi_{k}} \frac{\partial D^{i} v}{\partial x_{j}}+\sum_{l, p=0} \varepsilon^{l+p-1} \sum_{|i|=l} \frac{\partial}{\partial \xi_{j}}\left(A_{k j} N^{p, i}\right) \frac{\partial D^{i} v}{\partial x_{k}} \\
& +\sum_{l, p=0} \varepsilon^{l} \sum_{|i|=l} A_{k j} N^{p, i} \frac{\partial^{2} D^{i} v}{\partial x_{k} \partial x_{j}} .
\end{aligned}
$$

Next, we shift indices in the sums above to get

$$
\begin{aligned}
\sum_{l, p=0} \varepsilon^{l+p} \sum_{|i|=l}-\omega^{2} \rho N^{p, i} D^{i} v & =\sum_{l=0, p=2} \varepsilon^{l+p-2} \sum_{|i|=l}-\omega^{2} \rho N^{p-2, i} D^{i} v \\
\sum_{l, p=0} \varepsilon^{l+p-1} \sum_{|i|=l} A_{k} \frac{\partial N^{p, i}}{\partial \xi_{k}} \frac{\partial D^{i} v}{\partial x_{j}} & =\sum_{l=1, p=0} \varepsilon^{l+p-2} \sum_{|i|=l} A_{k i_{1}} \frac{\partial N^{p, i_{2} \cdots i_{1}}}{\partial \xi_{k}} D^{i} v \\
\sum_{l, p=0} \varepsilon^{l+p-1} \sum_{|i|=l} \frac{\partial}{\partial \xi_{j}}\left(A_{k j} N^{p, i}\right) \frac{\partial D^{i} v}{\partial x_{k}} & =\sum_{l=1, p=0} \varepsilon^{l+p-2} \sum_{|i|=l} \frac{\partial}{\partial \xi_{j}}\left(A_{i_{1}} N^{p, i_{2} \cdots i_{1}}\right) D^{i} v \\
\sum_{l, p=0} \varepsilon^{l} \sum_{|i|=l} A_{k j} N^{p, i} \frac{\partial^{2} D^{i} v}{\partial x_{k} \partial x_{j}} & =\sum_{l=2, p=0} \varepsilon^{p+l-2} \sum_{|i|=l} A_{i_{1} i_{2}} N^{p, i_{3} \cdots i_{1}} D^{i} v .
\end{aligned}
$$


Then (3.6) transforms into

$$
f \sim \sum_{l, p=0} \varepsilon^{l+p-2} \sum_{|i|=l} H^{p, i}(\xi) D^{i} v
$$

where $H^{p, i}$ depend on $N^{p, i}, A_{k j}, \omega$ and $\rho$. Since the left-hand side is of order $\varepsilon^{0}$, we obtain

$$
N^{0,0}=N^{1,0}=I,
$$

where $I$ denotes the unit matrix. Also, we assume that $N^{p, i}=0$ if at least one of $p$ and $|i|$ is negative. Then, collecting terms in (3.7), we have for $H^{p, i}$ the expressions

$$
\begin{aligned}
& H^{0,0}=0 \\
& H^{1,0}=L_{\xi} N^{1,0}=0 \\
& H^{p, 0}=-\omega^{2} \rho N^{p-2,0}+L_{\xi} N^{p, 0} \quad(p \geq 2) \\
& H^{0, i_{1}}=\frac{\partial A_{i_{1} j}}{\partial \xi_{j}}+L_{\xi} N^{0, i_{1}} \quad \text { and } \quad H^{1, i_{1}}=\frac{\partial A_{i_{1} j}}{\partial \xi_{j}}+L_{\xi} N^{1, i_{1}}
\end{aligned}
$$

and

$$
\begin{aligned}
H^{p, i}= & -\omega^{2} \rho N^{p-2, i}+A_{k i_{1}} \frac{\partial N^{p, i_{2} \cdots i_{i}}}{\partial \xi_{k}} \\
& +\frac{\partial}{\partial \xi_{j}}\left(A_{i_{1} j} N^{p, i_{2} \cdots i_{1}}\right)+A_{i_{1} i_{2}} N^{p, i_{3} \cdots i_{1}}+L_{\xi} N^{p, i} .
\end{aligned} \quad(p \geq 0,|i|>1)
$$

If we require that $H^{p, i}$ be constant, equations (3.8) and (3.9) can be used to determine $N^{p, i}$ recursively.

All equations above are of the form

$$
L_{\xi} N^{p, i}=-T^{p, i}-H^{p, i}
$$

where

$$
T^{p, i}=-\omega^{2} \rho N^{p-2, i}+A_{k i_{1}} \frac{\partial N^{p, i_{2} \cdots i_{i}}}{\partial \xi_{k}}+\frac{\partial}{\partial \xi_{j}}\left(A_{i_{1} j} N^{p, i_{2} \cdots i_{1}}\right)+A_{i_{1} i_{2}} N^{p, i_{3} \cdots i_{1}} .
$$

Note that $T^{p, i}$ depends on the previously obtained $N^{p^{\prime}, i^{\prime}}$ with $p^{\prime}+\left|i^{\prime}\right|<p+|i|$. We specify the constants $H^{p, i}$ to be $\left\langle T^{p, i}\right\rangle$, and write

$$
L_{\xi} N^{p, l}=-T^{p, i}+\left\langle T^{p, i}\right\rangle .
$$

This choice of $H^{p, i}$ guarantees that each cell problem is uniquely solvable up to a constant matrix. To show this, consider the variational formulation of cell problems (3.10). Denote by $V^{1}$ and $V^{0}$ the spaces of 1-periodic vector functions

$$
V^{1}=\left\{v \in H_{\text {loc }}^{1}:\langle v\rangle=0\right\} \quad \text { and } \quad V^{0}=\left\{v \in L_{l o c}^{2}:\langle v\rangle=0\right\} .
$$

Consider a sesquilinear form $b$ on $V^{1}$ given by

$$
b(u, v)=\int B(u, v) d x, \quad \text { where } B(u, v)(x)=A_{k j}^{i l} \frac{\partial u_{i}}{\partial x_{k}} \frac{\partial \bar{v}_{l}}{\partial x_{j}} .
$$

The variational formulation of a cell problem now reads: find $u \in V^{1}$ such that

$$
b(u, v)=\langle f, v\rangle
$$

for some $f \in V^{0}$ and all $v \in V^{1}$. Of course, the actual cell problem (3.10) is a matrix. one, so we have to solve several vector problems and determine columns of an unknown matrix one by one. 
Theorem 3.1. There exists a unique solution of problem (3.11).

Proof. We note first that the symmetry conditions for $A_{k j}$ remain the same as in the real-valued case. This implies $B(u, u)=e(u)^{*} A e(u)$, where $A$ is the fourth order tensor with components $A_{k j}^{(i l)}$ and $e(u)=\frac{1}{2}\left(\frac{\partial u_{i}}{\partial x_{j}}+\frac{\partial u_{j}}{\partial x_{i}}\right)$. This and the ellipticity condition for $A$ implies $|B(u, u)| \geq c_{1}\|e(u)\|_{L^{2}(Q)}^{2}$. By [13: Theorem 2.8], the second Korn inequality $\|u\|_{H^{1}(Q)} \leq C\|e(u)\|_{L^{2}(Q)}$ holds for all $u \in \dot{V}^{1}$. Hence, the form $B$ is coercive on $V^{1}$, and by the Lax-Milgram lemma, problem (3.11) is uniquely solvable for any $f \in V^{0}(Q)$

The asymptotic series for $u$ now takes the form

$$
\begin{aligned}
u \sim & H^{2,0} v(x)+H^{i_{1}, i_{2}} \frac{\partial^{2} v(x)}{\partial x_{i_{1}} \partial x_{i_{2}}}+\sum_{p+l>2} \varepsilon^{l+p-2} \sum_{|i|=l} H^{p, i} D^{i} v \\
= & -\omega^{2}\langle\rho\rangle v(x)+\sum_{i_{1}, i_{2}=1}^{n}\left\langle A_{k i_{1}} \frac{\partial N^{0, i_{2}}}{\partial \xi_{k}}+A_{i_{1} i_{2}}\right\rangle \frac{\partial^{2} v(x)}{\partial x_{i_{1}} \partial x_{i_{2}}} \\
& +\sum_{p+l>2} \varepsilon^{l+p-2} \sum_{|i|=l} H^{p, i} D^{i} v .
\end{aligned}
$$

Representing $v$ as an asymptotic series $v(x) \sim \sum_{q=0} \varepsilon^{q} v_{q}(x)$ we obtain a chain of averaged problems for successive determination of $v_{q}$ :

$$
\hat{L} v_{q}=f_{q}
$$

where

$$
\hat{L} v=-\omega^{2}\langle\rho\rangle u+\sum_{i_{1}, i_{2}=1}^{n}\left\langle A_{k i_{1}} \frac{\partial N^{0, i_{2}}}{\partial \xi_{k \cdot}}+A_{i_{1} i_{2}}\right\rangle \frac{\partial^{2} v(x)}{\partial x_{i_{1}} \partial x_{i_{2}}}
$$

and

$$
\begin{aligned}
& f_{0}=f \\
& f_{1}=-\sum_{l+p=3} \sum_{|i|=l} H^{p, i} D^{i} v_{0} \\
& f_{2}=-\sum_{l+p=4} \sum_{|i|=l} H^{p, i} D^{i} v_{0}-\sum_{l+p=3} \sum_{|i|=l} H^{p, i} D^{i} v_{1},
\end{aligned}
$$

and, generally,

$$
f_{q}=-\sum_{t=0}^{q-1} \sum_{l+p=q-t+2} \sum_{|i|=l} H^{p, i} D^{i} v_{t}
$$

The first equation in chain (3.12) is the homogenized system

$$
-\omega^{2} \rho u+\sum_{i_{1}, i_{2}=1}^{n}\left\langle A_{k i_{1}} \frac{\partial N^{0, i_{2}}}{\partial \xi_{k}}+A_{i_{1} i_{2}}\right\rangle \frac{\partial^{2} v(x)}{\partial x_{i_{1}} \partial x_{i_{2}}}=f .
$$


The matrices $N^{0, i_{2}}$ above are obtained as solutions of the cell problem

$$
L_{\xi} N^{0, i_{2}}=-\frac{\partial}{\partial \xi_{j}} A_{i_{2} j}
$$

satisfying the periodic boundary conditions and the transmission conditions $\left[N^{0, i_{2}}\right]=0$ and $\left[A_{k j} \frac{\partial N^{p, i}}{\partial \xi_{k}} n\right]=0$ on the interface hypersurface $S$.

\section{Interface matching and boundary layers}

In the previous section we did not consider boundary conditions, so the construction above applies only locally in $\mathbf{R}^{n}$. To investigate the nature of the changes needed to incorporate boundary effects, consider the following model problem. Suppose that the plane interface $\left\{x: x_{n}=0\right\}$ separates two different periodic media. We assume that equations (3.1) together with the constitutive relations (3.2) with possibly different 1periodic matrices $A_{k j}^{ \pm}$are valid in the halfspaces $K^{+}=\left\{x: x_{n}>0\right\}$ and $K^{-}=\{x$ : $x_{n}<0$ \}, respectively. A particular case of this is acoustics in a two-layer media of the type homogeneous fluid above, fluid-saturated sediment below. At this point we prescribe no conditions on $u$ as $\left|x_{n}\right| \rightarrow \infty$. Our primary interest is to investigate how the presence of the interface affects homogenization.

For any $z=\left(z_{1}, \ldots z_{n}\right) \in \mathbf{R}^{n}$, let $\hat{z}$ denote the vector $\left(z_{1}, \ldots z_{n-1}, 0\right)$. In what follows, we use the notations

$$
\begin{aligned}
\omega(a, b) & =\left\{x: a<x_{n}<b\right\} \\
\hat{\omega}(a, b) & =\left\{x: 0<x_{j}<1(j=1, \ldots, n-1) \text { and } a<x_{n}<b\right\} \\
\Gamma_{t} & =\left\{x: x_{n}=t\right\}
\end{aligned}
$$

with $\hat{\Gamma}_{t}$ modified accordingly. We also denote

$$
\begin{aligned}
& Q=\left\{x: x_{j} \in(0,1)(j=1, \ldots, n)\right\} \\
& \hat{Q}=\left\{x: x_{j} \in(0,1)(j=1, \ldots, n-1) \text { and } x_{n}=0\right\}
\end{aligned}
$$

Denote by $\hat{H}^{1}(\omega(a, b))$ the space of locally $H^{1}$-functions 1 -periodic in $\hat{x}$. We recall that $L$ denotes the differential operator in equations (3.1). A function $u \in \hat{H}^{1}(\omega(a, b))$ is a weak periodic in $\hat{x}$ solution of the problem:

$$
L u=f
$$

in $\omega(a, b)$ if for any $v \in \hat{H}^{1}(\omega(a, b))$ such that $v=0$ on $\Gamma_{a} \cup \Gamma_{b}$ the relation

$$
\int_{\dot{\omega}(a, b)}\left(A^{h k} \partial_{k} u, \partial_{h} v\right) d x=\int_{\dot{\omega}(a, b)}(f, v) d x
$$

holds. 
Below quantitics with sub- or superscript + are defined in $K^{+}$, and similarly refers to a quantity defined in $K^{-}$. In $K^{+}$, we look for asymptotic expansions of the form

$$
u_{+} \sim \sum_{p, l=0}^{\infty} \varepsilon^{p+l} \sum_{|i|=l}\left(N_{+}^{p, i}+M_{+}^{p, i}\right)(\xi) D^{i} v_{+}(x)+S_{+}^{p, i} D^{i} v_{-}(x)
$$

where $v_{ \pm}$are asymptotic series formed by (so far) arbitrary solutions of the chain of homogenized problems (3.12) in $K^{ \pm}$. In $K^{-}$we look for a similar expansion with all pluses replaced by minuses and vice versa. The matrices $N^{p, i}$ are as above, and $M_{+}^{p, i}$ and $S_{+}^{p, i}$ are matrices 1-periodic in $\hat{\xi}$. Substituting (4.1) into the original equations (3.1) and repeating the calculations of Section 3 , we obtain the identical equations for $M_{+}^{p, i}$ and $S_{+}^{p, i}$, written explicitly only for $M_{+}^{p, i}$ :

$$
L_{\xi}^{+} M_{+}^{p, i}=M M_{+}^{p, i}
$$

in $K^{+}$, where $M M_{+}^{p, i}$ are of the form

$$
M M_{+}^{p, i}=M M_{+, 0}^{p, i}+\frac{\partial M M_{+, j}^{p, i}}{\partial \xi_{j}}
$$

wherc

$$
\begin{aligned}
& M M_{+, 0}^{p, i}=-\omega^{2} \rho M_{+}^{p-2, i}+A_{i_{1} i_{2}}^{+} M_{+}^{p, i_{3} \cdots i_{l}} \\
& M M_{+, j}^{p, i}=A_{j i_{1}}^{+} M_{+}^{p, i_{1} \ldots l_{l}}+A_{i_{1} j}^{+} M_{+}^{p, i_{2} \cdots i_{l}} .
\end{aligned}
$$

To start the chain, we set $M_{+}^{0,0}=M_{+}^{1,0}=S_{+}^{0,0}=S_{+}^{1,0}=0$. On the interface $x_{n}=0$ we impose the transmission conditions

$$
\left.\begin{array}{rl}
u^{+} & =u^{-} \\
\sigma^{+}\left(u^{+}\right)_{n} & =\sigma^{-}\left(u^{-}\right)_{n}
\end{array}\right\}
$$

where

$$
\sigma^{ \pm}\left(u^{ \pm}\right)_{n}=A_{ \pm}^{n j} \frac{\partial}{\partial x_{j}} u^{ \pm}
$$

These conditions arise due to requirements of continuity of displacements and stresses. Differentiating $u^{ \pm}$and shifting indices in the sums in the same fashion as in Section 3, we obtain

$$
\begin{aligned}
\sigma^{+}\left(u^{+}\right)_{N}= & \sum_{p, l=0}^{\infty} \varepsilon^{p+l-1} \\
& \times \sum_{|i|=l}\left[A_{n j}^{+} \frac{\partial}{\partial \xi_{j}}\left(N_{+}^{p, i}+M_{+}^{p, i}\right)+A_{n i_{1}}^{+}\left(N_{+}^{p, i_{2} \cdots i_{l}}+M_{+}^{p, i_{2} \cdots i_{l}}\right)\right] D^{i} v^{+} \\
& +\sum_{p, l=0}^{\infty} \varepsilon^{p+l-1} \sum_{|i|=l}\left[A_{n j}^{+} \frac{\partial S_{+}^{p, i}}{\partial \xi_{j}}+A_{n i_{1}}^{+} S_{+}^{p, i_{2} \cdots i_{l}}\right] D^{i} v^{-}
\end{aligned}
$$


and a similar expression for $\sigma^{-}\left(u^{-}\right)_{n}$. Substituting into the second equation in (4.3), collecting terms and combining with equations (4.2) we obtain the following transmission problems for determination of the pair of matrices $M_{+}^{p, i}$ and $S_{-}^{p, i}$ :

$$
\left.\begin{array}{rl}
L_{\xi}^{+} M_{+}^{p, i}=M M_{+}^{p, i} & \text { in } K^{+} \\
L_{\xi}^{-} S_{-}^{p, i}=S S_{-}^{p, i} & \text { in } K^{-}
\end{array}\right\}
$$

with the interface conditions

$$
N_{+}^{p, i}+M_{+}^{p, i}=S_{-}^{p, i}+k_{+}^{p, i}
$$

and

$$
A_{n j}^{+} \frac{\partial}{\partial \xi_{j}}\left(N_{+}^{p, i}+M_{+}^{p, i}\right)+A_{n i_{1}}^{+}\left(N_{+}^{p, i_{2} \cdots i_{1}}+M_{+}^{p, i_{2} \cdots i_{i}}\right)=A_{n j}^{-} \frac{\partial S_{-}^{p, i}}{\partial \xi_{j}}+A_{n i_{1}}^{-} S_{-}^{p, i_{2} \cdots i_{1}}+t_{+}^{p, i}
$$

where $k_{+}^{p, i}$ and $t_{+}^{p, i}$ are constant matrices. We look for solution of this problem in the class of 1-periodic in $\hat{\xi}$ matrices which decay exponentially as $\left|\xi_{n}\right| \rightarrow \infty$. Similarly, the pair $M_{-}^{p, i}, S_{+}^{p, i}$ should be a solution to the problem

$$
\left.\begin{array}{cc}
L_{\xi}^{-} M_{-}^{p, i}=M M_{-}^{p, i} & \text { in } K^{-} \\
L_{\xi}^{+} S_{+}^{p, i}=S S_{+}^{p, i} & \text { in } K^{+}
\end{array}\right\}
$$

with the interface conditions

$$
N_{-}^{p, i}+M_{-}^{p, i}=S_{+}^{p, i}+k_{-}^{p, i}
$$

and

$$
A_{n j}^{-} \frac{\partial}{\partial \xi_{j}}\left(N_{-}^{p, i}+M_{-}^{p, i}\right)+A_{n i_{1}}^{-}\left(N_{-}^{p, i_{2} \cdots i_{1}}+M_{-}^{p, i_{2} \cdots i_{l}}\right)=A_{n j}^{+} \frac{\partial S_{+}^{p, i}}{\partial \xi_{j}}+A_{n i_{1}}^{+} S_{+}^{p, i_{2} \ldots i_{1}}+t_{-}^{p, i}
$$

at the interface. Let us define the operator $L$ to be $L^{+}$in $K^{+}$and $L^{-}$in $K^{-}$. Then the problems above can be written in the common form

$$
\begin{aligned}
L u & =f & & \text { in } K^{+} \cup K^{-} \\
{[u] } & =\Phi(\hat{x}) & & \text { at } x_{n}=0 \\
{\left[\sigma(u)_{n}\right] } & =\Psi(\hat{x})+t & & \text { at } x_{n}=0 .
\end{aligned}
$$

In order to formulate the solvability theorem, we first introduce some definitions.

Definition 4.1. Let $u\left(\hat{x}, x_{n}\right) \in L_{\text {loc }}^{2}$ be a vector function 1 -periodic in $\hat{x}$. We say that $u$ has one-sided exponential decay, if the estimate

$$
\|u\|_{L^{2}\left(Q_{0}\right)} \leq C e^{-a|s|}
$$

holds either for $s \in \mathbf{Z}^{+}$or $s \in \mathbf{Z}^{-}$, with constants $C>0$ and $a>0$ independent of $s$. If (4.9) holds for all $s \in \bar{Z}$, we will say that $u$ has two-sided exponential decay.

To describe the behavior at infinity we will use 
Definition 4.2. A vector function $u$ will be called one-sided exponentially stabilizing if there exist a constant vector $w$ such that the function $u-w$ satisfies estimate (4.9) either for $s \in \mathrm{Z}^{+}$or $s \in \mathrm{Z}^{-}$. If there is a pair of constant vectors $w^{+}$and $w^{-}$ such that one-sided estimates (4.9) hold for both respective differences, we will call $u$ two-sided exponentially stabilizing to $w^{+}$and $w^{-}$.

Theorem 4.1. Suppose that $f$ in (4.8) has two-sided exponential decay. Then there exist constant vectors $t, w^{+}, w^{-}$such that problem (4.8) has a 1-periodic in $\xi$ solution $u$ such that $e(u)$ has two-sided exponential decay, and $u$ is two-sided exponentially stabilizing to $w^{+}$and $w^{-}$. Moreover,

$$
t=\int_{K^{-}} f(x) d x-\int_{K^{+}} f(x) d x-\int_{\hat{Q}} \Psi(\hat{x}) d \hat{x} .
$$

Proof. First, we prove the theorem under the assumption that $A^{j k}, f, \Phi, \Psi$ are smooth functions of their arguments. Consider a vector function $U$ defined in $K^{+}$and satisfying the following conditions:

i) $U(\hat{x}, 0)=-\Phi(\hat{x})$.

ii) $\sigma(U)_{n}(\hat{x}, 0)=-\Psi(\hat{x})-t$.

iii) $U$ and $e(U)$ have one-sided exponential decay.

The existence of $U$ follows from Borel's theorem (see, for instance, [8: Theorem 1.2.6]). Next, consider the function $v=u-U$, and let $\tilde{F}$ denote the function equal to $f-F$ in $K^{+}$, and equal to $f$ in $K^{-}$. The function $v$ is a solution to the problem

$$
\left.\begin{array}{rl}
L v=\tilde{F} & \text { in } K^{+} \cup K^{-} \\
{[v]=0} & \text { when } x_{n}=0 \\
\left.v\rangle_{n}\right]=0 & \text { when } x_{n}=0 .
\end{array}\right\}
$$

In other words, $v$ must be a global solution of the system of equations in (4.11). By Theorem 5.4 from Section 5, for any constant vector $q$ this system has a solution $v$ such that $P(v, 0)=q$, where $P(v, 0)$ denotes a generalized moment of $v$ at $x_{n}=0$. For the definition and properties of momenta, we refer to Section 5 below, and to [12]. Theorem 5.4 also implies that $v$ satisfies the a priori estimate

$$
\|e(v)\|_{L^{2}(\dot{\omega}(-k, k))}^{2} \leq C\left(M e^{\left(A-\delta_{1}\right)|k|}+|k|\left\|q-\int_{\hat{\Gamma}_{0}} f_{n} d \hat{x}\right\|^{2}\right) .
$$

Now apply Theorem 5.3 from Section 5. We see that $v$ is exponentially stabilizing in $K^{+}$if $P(v, 0)=-\int_{K^{-}} f(x) d x$. Also, $v$ will be exponentially stabilizing in $K^{-}$ provided $P(v, 0)=-\int_{K^{-}} f(x) d x$. Combined, these equalities imply that $v$ will be two-sided exponentially stabilizing if we set

$$
P(v, 0)=-\int_{K^{-}} f(x) d x=-\int_{K^{-}} f(x) d x .
$$


Hence we obtain that

$$
\int_{K^{+}} F(x) d x=\int_{K^{+}} f(x) d x-\int_{K^{-}} f(x) d x
$$

Consider the left-hand side separately. Since $F=\operatorname{div}(\sigma(U))$, we can integrate by parts. Due to periodicity, contributions of the derivatives with respect to $\hat{x}_{j}$ vanish. Hence,

$$
\int_{K^{+}} F(x) d x=\int_{0}^{\infty} \partial_{x} \int_{\hat{Q}} \sigma(U)_{n} d \hat{x} d x_{n}
$$

The choice of $U$ makes the contribution of the upper limit in the $x_{n}$-integral zero, and we obtain

$$
\int_{K^{+}} F(x) d x=-\int_{\dot{Q}} \sigma(U)_{n}(\hat{x}, 0)=-\int_{\dot{Q}} \Psi(\hat{x}) d \hat{x} .
$$

Combining this with (4.12) we obtain (4.10). The existence of the constant vectors $w^{+}$ and $w^{-}$as well as two-sided exponential decay of $e(v)$ follow from Theorem 5.3. Now, using condition iii) for $U$ we find that $u$ is two-sided exponentially stabilizing to $w^{+}$and $w^{-}$, and that $e(u)$ has two-sided exponential decay.

Finally, since constants in the estimates depend only on dimension and bounds for coefficient matrices, we can approximate the actual $A^{j k}, f, \Phi$ and $\Psi$ by sequences of smooth functions and pass to the limit in the estimates

Once $M^{p, i}$ and $S^{p, i}$ are found, we can obtain a sequence of macroscopic transmission problems. We use the notation $v_{q}(x)^{ \pm}, L^{ \pm}$and so on to refer to vectors and operators defined in $K^{+}$and $K^{-}$, respectively. Using these notations and the above transmission conditions for $M^{p, i}$ and $S^{p, i}$, we can write

$$
[u] \sim \sum_{p, l=0}^{\infty} \varepsilon^{p+l} \sum_{|i|=l} k_{+}^{p, i} D^{i} v_{+}(x)-k_{-}^{p, i} D^{i} v_{-}(x) .
$$

Similarly, the jump of the normal stress at the interface can be written as

$$
\left[\sigma(u)_{n}\right] \sim \sum_{p, l=0}^{\infty} \varepsilon^{p+l-1} \sum_{|i|=l} t_{+}^{p, i} D^{i} v_{+}(x)-t_{-}^{p, i} D^{i} v_{-}(x)
$$

In order to satisfy the original continuity requirements, we need expressions on the right of these equations to be zero. Now, representing $v(x)$ as an asymptotic series $v=\sum \varepsilon^{q} v_{q}$ and collecting terms in (4.13) and (4.14) we obtain the chain of transmission problems

$$
\hat{L}^{ \pm} v_{q}^{ \pm}=f_{q}^{ \pm}
$$

in $K^{ \pm}$, where the homogenized operators $\hat{L}^{ \pm}$and the right-hand sides $f_{q}$ are defined as in Section 3. On the interface $x_{n}=0$ the transmission conditions

$$
\begin{aligned}
{\left[k^{0,0} v_{q}\right] } & =\sum_{1+p+k=q, k<q|i|=1} \sum_{1+1} k_{+}^{p, i} D^{i} v_{+}(x)-k_{-}^{p, i} D^{i} v_{-}(x) \\
{\left[t^{0, i_{1}} D^{i_{1}} v_{q}\right] } & =\sum_{1+p+k-1=q, k<q|i|=1} \sum_{+} t_{+i}^{p, i} D^{i} v_{+}(x)-t_{-}^{p, i} D^{i} v_{-}(x)
\end{aligned}
$$


are fulfilled.

Let us now compute explicitly transmission conditions for the first two problems in this chain. Recall that $N^{0,0}=I$ and $M^{0,0}=S^{0,0}=0$. This, together with (4.5) and (4.7) immediately gives

$$
k_{+}^{0,0}=k_{-}^{0,0}=I \quad \text { and } \quad t_{+}^{0,0}=t_{-}^{0,0}=t_{+}^{1,0}=t_{-}^{1,0}=0 .
$$

Next, consider equations (4.15), (4.16) for $p=0$ and some fixed $l=i_{1}$. The first observation is that $M M^{0, i_{1}}=S S^{0, i_{1}}=0$ which yields $k_{ \pm}^{0, i_{1}}=0$ after applications of Theorem 5.3. Next, we apply the formula for $t$ from Theorem 4.1 to get

$$
t_{+}^{0, i_{1}}=\int_{\dot{Q}}\left(A_{n j}^{+} \frac{\partial N_{+}^{p, i_{1}}}{\partial \xi_{j}}+A_{n i_{1}}^{+}\right) d \hat{x} .
$$

Let $\hat{A}_{n j}$ denote the homogenized matrices

$$
\hat{A}_{n i_{1}}=\int_{Q}\left(A_{n j} \frac{\partial N^{0, i_{1}}}{\partial \xi_{j}}+A_{n i_{1}}\right) d x
$$

Moreover, denote

$$
\tilde{A}_{n i_{1}}=A_{n j} \frac{\partial N^{0, i_{1}}}{\partial \xi_{j}}+A_{n i_{1}}-\hat{A}_{n i_{1}}
$$

Now we can write (4.17) as

$$
t_{+}^{0, i_{1}}=\hat{A}_{n i_{1}}^{+}+\int_{\dot{Q}} \tilde{A}_{n i_{1}}^{+} d \hat{x}
$$

We want to show that the integral is zero. Note that the cell system for $N_{+}^{0, i_{1}}$ can be written as $\frac{\partial}{\partial \xi_{k}} \tilde{A}_{k i_{1}}^{+}=0$. If $\tilde{A}_{k j}$ are smooth, we can integrate this equation from zero to $t<1$ with respect to $x_{n}$, and then integrate over $\hat{Q}$. The integrals containing $A_{k i_{1}}^{+}$with $k \neq n$ vanish because of periodicity, and we get

$$
\int_{\dot{Q}} \tilde{A}_{n i_{1}}^{+}(t, \hat{x}) d \hat{x}=\int_{\dot{Q}} \tilde{A}_{n i_{1}}^{+}(0, \hat{x}) d \hat{x}
$$

for all $t \in[0,1]$. In other words, the integral on the right of $(4.18)$ is a constant. Integrating this constant on the interval $(0,1)$, we will obtain the average of $\tilde{A}_{n i_{1}}^{+}$which is zero by the definition of $\tilde{A}_{n i_{1}}^{+}$. Hence the original constant must be zero. In the case of a general $\tilde{A}_{n i_{1}}^{+}$the same conclusion is obtained by approximating $\tilde{A}_{n i_{1}}^{+}$by a sequence of smooth matrices and passing to the limit. Putting everything together we obtain

Proposition 4.1. The first non-trivial homogenized problem in the chain has the form $\hat{L} v_{0}=f$ in $K^{+} \cup K^{-}$, with the transmission conditions $\left[v_{0}\right]=0$ and $\left[\hat{A}_{n i_{1}} D^{i_{1}} v_{0}\right]=$ 0 at the interface $x_{n}=0$.

This shows that microstructure does not affect homogenized transmission conditions at this level. 


\section{A priori estimates of Saint-Venant type}

In this section the technical results needed to construct boundary layers are collected. The most important part here is an a priori estimate of Saint-Venant type obtained in Theorem 5.2. The importance of estimates of this type for investigation of behavior of solutions at infinity and proving existence theorems in unbounded domains has been recognized by Oleinik and Yosifian [11] in the context of linear elasticity. The development here follows closely that in the book [12: Chapter II/Sections 7 and 8]. In the present case, we need estimates for the system of viscoelasticity ( complex-valued coefficients are allowed). Moreover, we need to treat the transmission problem rather than Dirichlet or Neumann ones. This makes it necessary to derive estimates valid on both sides of the interface.

Recall that a function $u \in \hat{H}^{1}(\omega(a, b))$ is a weak periodic in $\hat{x}$ solution of the problem

$$
L u=f \quad \text { in } \omega(a, b)
$$

if for any $v \in \hat{H}^{1}(\omega(a, b))$ such that $v=0$ on $\Gamma_{a} \cup \Gamma_{b}$ the relation

$$
\int_{\dot{\sim}(a, b)}\left(A^{h k} \partial_{k} u, \partial_{h} v\right) d x=\int_{\dot{\omega}(a, b)}(f, v) d x
$$

holds. Next, we introduce generalized momenta $P(t, u)$ defined by

$$
P(t, u)=\lim _{s \rightarrow 0^{+}} \int_{\dot{\omega}(t, t+s)} A^{n k} \partial_{k} u d x
$$

The existence of momenta is proved in [12]. Moreover, we have

$$
P\left(t_{1}, u\right)-P\left(t_{2}, u\right)=\int_{\dot{\hat{u}}\left(t_{2}, t_{1}\right)} f d x
$$

for $b>t_{1}>t_{2}>b$, and if $A^{h k}$ and $f$ are sufficiently smooth, then

$$
P(t, u)=\int_{\hat{\Gamma}_{t}} A^{n k} \partial_{k} u d \hat{x}
$$

The following theorem is a basic version of the Saint-Venant principle slightly modified from [12].

Theorem 5.1. Let $s>h>0$ be integers, and let $u$ be a periodic in $\hat{x}$ solution of $L u=0$ in $\omega(s-h, s+h+1)$. Suppose that $P(s-1, u)=0$. Then

$$
\int_{\dot{\omega}(s, s+1)}|B(u, u)| d x \leq e^{-A h} \int_{\dot{\omega}(s-h, s+h+1)}|B(u, u)| d x,
$$

where $A$ is a positive constant independent of $s$ and $h$. 


\section{Proof. Denote}

$$
g=\hat{\omega}(s-h, s+1+h), \quad g^{1}=\hat{\omega}(s-h, s), \quad g^{2}=\hat{\omega}(s+1, s+1+h) .
$$

Pick a sequence $u^{m}$ of smooth functions converging to $u$. Then define a function $\Phi\left(x_{n}\right)$ by

$$
\Phi\left(x_{n}\right)= \begin{cases}\exp \left(A\left[x_{n}-(s-h)\right]\right. & \text { for } x \in g^{1} \\ \exp \left(A\left[s+1+h-x_{n}\right]\right) & \text { for } x \in g^{2} \\ \exp (A h) & \text { for all other } x \in g .\end{cases}
$$

Here $A$ is a positive constant to be specified. Then choose a test function $v=(\Phi-1) u^{m}$ and plug into the integral identity. We obtain

$$
\begin{aligned}
\int_{g}\left(A^{h k} \partial_{k} u,(\Phi-1) \partial_{h} u^{m}\right) d x & =-\int_{g}\left(A^{h k} \partial_{k} u, \partial_{h} \Phi u^{m}\right) d x \\
& =-\int_{g^{1} \cup g^{2}}\left(A^{n k} \partial_{k} u, \partial_{n} \Phi u^{m}\right) d x
\end{aligned}
$$

Next, we write

$$
g^{1}=\bigcup_{d=0}^{h-1} w_{d} \quad \text { where } w_{d}=\left\{x: s-h+d<x_{n}<s-h+d+1\right\} .
$$

Fix $d$ temporarily and choose a constant vector $C$ such that $\int_{w_{d}}\left(u^{m}+C\right) d x=0$. Then, using the second Korn inequality and the estimate

$$
\|e(u)\|_{L^{2}\left(w_{k}\right)} \leq c\|B(u, u)\|_{L^{2}\left(w_{k}\right)},
$$

we obtain

$$
\left\|\left(u^{m}+C\right)\right\|_{L^{2}\left(w_{k}\right)} \leq M\left\|B\left(u^{m_{z}}, u^{m}\right)\right\|_{L^{2}\left(w_{k}\right)},
$$

where $M$ is independent of $m$ and $d$. Next we observe that

$$
\int_{w_{k}}\left(A^{n k} \partial_{k} u, \partial_{n} \Phi C\right) d x=\int \partial_{n} \Phi \int\left(A^{n k} \partial_{k} u, C\right) d \hat{x} d x_{n}=0,
$$

since $P(s+1, u)=0$ implies $P(t, u)=0$ for all $s-h<t<s+1+h$. Hence,

$$
\begin{aligned}
\mid \int_{w_{k}} & \left(A^{n k} \partial_{k} u, \partial_{n} \Phi u^{m}\right) d x \mid \\
\quad & \left|\int_{w_{k}}\left(A^{n k} \partial_{k} u, \partial_{n} \Phi\left(u^{m}+C\right)\right) d x\right| \\
& =\left|\int_{w_{k}}\left(A^{n k} \partial_{k} u, A \Phi\left(u^{m}+C\right)\right) d x\right| \\
& \leq C_{1} M A e^{A(s-h+d+1)}\left(\int_{w_{k}}|B(u, u)| d x\right)^{\frac{1}{2}}\left(\int_{w_{k}}\left|B\left(u^{m}, u^{m}\right)\right| d x\right)^{\frac{1}{2}} \\
& \leq C_{2} M A e^{A} \int_{w_{k}}|B(u, u)| \Phi d x+R_{m},
\end{aligned}
$$


where $R_{m} \rightarrow 0$ as $m \rightarrow \infty$. Summing over all $w_{k}$ we obtain

$$
\left|\int_{g^{1}}\left(A^{n k} \partial_{k} u, \partial_{n} \Phi u^{m}\right) d x\right| \leq C_{3} M A e^{A} \int_{g^{1}}|B(u, u)| \Phi d x+h R_{m} .
$$

Repeating the argument we obtain a similar estimate for $g^{2}$. Finally, let $m \rightarrow \infty$. Then

$$
\int_{g}|B(u, u)|(\Phi-1) d x \leq C_{4} M A e^{A} \int_{g^{1} \cup g^{2}}|B(u, u)| \Phi d x .
$$

Choose $A$ so that the constant on the right is equal to one. Then

$$
\int_{g \backslash\left(g^{1} \cup g^{2}\right)}|B(u, u)| \Phi d x \leq \int_{g}|B(u, u)| d x .
$$

Multiplying by $e^{-A h}$ we obtain the estimate desired

Next, we need to generalize this to the case when $f$ and $P(s-h, u)$ are non-zero. The prototype of the main estimate is given by the

Lemma 5.1. Let $N$ be a positive integer. The system

$$
L U=f_{0}+\partial_{i} f_{i} \quad \text { in } \omega(-N, N)
$$

with boundary conditions

$$
\sigma(U)_{n}= \begin{cases}\phi+f_{i} & \text { on } \Gamma_{-N} \\ \psi-f_{i} & \text { on } \Gamma_{N}\end{cases}
$$

satisfying the compatibility condition

$$
\int_{\dot{\Gamma}_{-N}} \phi(\hat{x}) d \hat{x}+\int_{\hat{\Gamma}_{N}} \psi(\hat{x}) d \hat{x}=\int_{\dot{\omega}(-N, N)} f(x) d x
$$

has a unique solution $U$ satisfying the estimate

$$
\|e(U)\|_{L^{2}(\dot{\omega}(-N, N))}^{2} \leq C\left[\sum_{i=0}^{n}\|f\|_{L^{2}(\dot{\omega}(-N, N))}^{2}+\sum_{m=-N}^{N}\left\|\psi_{m}\right\|_{L^{2}\left(\Gamma_{m}\right)}^{2}\right]
$$

where

$$
\left.\begin{array}{rl}
\psi_{-N} & =\phi \\
\psi_{m} & =\int_{\dot{\dot{\omega}(m, N)}} f(x) d x-\int_{\Gamma_{N}} \psi(\hat{x}) d \hat{x} \quad(m=-N+1, \ldots, N-1) \\
\psi_{N} & =-\psi .
\end{array}\right\}
$$

Proof. Consider the problem

$$
\left.\begin{array}{rlrl}
L V^{m} & =f & & \text { in } \omega(m-1, m) \\
\sigma\left(V^{m}\right) & =-\psi_{m} & & \text { on } \Gamma_{m} \\
\sigma\left(V^{m}\right) & =\psi_{m-1} & & \text { on } \Gamma_{m-1} .
\end{array}\right\}
$$


By [12: Theorem 6.3] modified slightly for the case of complex-valued coefficients, problem (5.7) is uniquely solvable if and only if the compatibility condition

$$
\int_{\bar{\Gamma}_{m-1}} \psi_{m-1}(\hat{x}) d \hat{x}-\int_{\Gamma_{m}} \psi_{m}(\hat{x}) d \hat{x}=\int_{\hat{\dot{\omega}(m-1, m)}} f(x) d x
$$

is satisfied. Let us check these conditions for problem (5.7). Using (5.6) we have

$$
\begin{aligned}
& \int_{\hat{\Gamma}_{m-1}} \psi_{m-1}(\hat{x}) d \hat{x}-\int_{\Gamma_{m}} \psi_{m}(\hat{x}) d \hat{x} \\
& \quad=-\int_{\dot{\Gamma}_{-N}} \psi_{m-1}(\hat{x}) d \hat{x}+\int_{\Gamma_{N}} \psi(\hat{x}) d \hat{x}+\int_{\hat{\omega}(m-1, N)} f(x) d x-\int_{\Gamma_{N}} \psi(\hat{x}) d \hat{x} \\
& \quad=\int_{\hat{\dot{\omega}}(m-1, m)} f(x) d x .
\end{aligned}
$$

Also, for $m=-N+1$ we get

$$
\begin{aligned}
& \int_{\hat{\Gamma}_{-N}} \phi(\hat{x}) d \hat{x}-\int_{\Gamma_{-N+1}} \psi_{-N+1}(\hat{x}) d \hat{x} \\
& \quad=\int_{\hat{\Gamma}_{-N}} \phi(\hat{x}) d \hat{x}-\int_{\dot{\omega}(-N+1, N)} f(x) d x+\int_{\hat{\Gamma}_{N}} \psi(\hat{x}) d \hat{x} \\
& \quad=\int_{\hat{\Gamma}_{-N}} \phi(\hat{x}) d \hat{x}+\int_{\hat{\Gamma}_{N}} \psi(\hat{x}) d \hat{x}-\int_{\dot{\omega}(-N, N)} f(x) d x+\int_{\dot{\omega}(-N,-N+1)} f(x) d x \\
& =\int_{\dot{\omega}(-N,-N+1)} f(x) d x
\end{aligned}
$$

and similarly, the condition also checks for $m=N$. By [12: Theorem 6.3] cited problems (5.7) are uniquely solvable. Moreover, the estimate

$$
\begin{aligned}
& \left\|e\left(V^{m}\right)\right\|_{L^{2}(\dot{\omega}(m-1, m))}^{2} \\
& \quad \leq C\left[\sum_{i=0}^{n}\left\|f_{i}\right\|_{L^{2}(\dot{\omega}(m-1, m))}^{2}+\left\|\psi_{m}\right\|_{L^{2}\left(\Gamma_{m}\right)}^{2}+\left\|\psi_{m-1}\right\|_{L^{2}\left(\Gamma_{m-1}\right)}^{2}\right]
\end{aligned}
$$

holds. Next, consider the sesquilinear forms

$$
\begin{aligned}
I_{m}= & -\int_{\dot{\omega}(m-1, m)} B\left(V^{m}, U\right) d x \\
= & \int_{\dot{\omega}(m-1, m)}\left(f_{0}, U\right) d x-\int_{\dot{\omega}(m-1, m)}\left(f_{i}, \partial_{i} U\right) d x \\
& +\int_{\Gamma_{m}}\left(\psi_{m}, U\right) d \hat{x}-\int_{\Gamma_{m-1}}\left(\psi_{m-1}, U\right) d \hat{x}
\end{aligned}
$$

Summing up we obtain

$$
\begin{aligned}
-\sum_{m=-N}^{N} I_{m}= & \int_{\dot{\omega}(-N, N)}(f, U) d x-\int_{\dot{\omega}(m-1, m)}\left(f_{i}, \partial_{i} U\right) d x \\
& +\int_{\Gamma_{-N}}(\phi, U) d \hat{x}-\int_{\Gamma_{N}}(\psi, U) d \hat{x}
\end{aligned}
$$


which shows that

$$
-\sum_{m=-N}^{N} I_{m}=\int_{\dot{\omega}(-N, N)} B(U, U) d x .
$$

Estimating the left-hand side in the last equation we get

$$
\begin{aligned}
\int_{\dot{\omega}(-N, N)} B(U, U) d x \\
\leq \sum_{m=-N}^{N} \int_{\dot{\omega}(m-1, m)}\left|B\left(V^{m}, U\right)\right| d x \\
\leq \sum_{m=-N}^{N}\left(\int_{\dot{\omega}(m-1, m)} B\left(V^{m}, V^{m}\right) d x\right)^{\frac{1}{2}}\left(\int_{\dot{\omega}(m-1, m)} B(U, U) d x\right)^{\frac{1}{2}} \\
\leq\left(\int_{\dot{\omega}(-N, N)} B(U, U) d x\right)^{\frac{1}{2}}\left(\sum_{m=-N}^{N} \int_{\dot{\omega}(m-1, m)} B\left(V^{m}, V^{m}\right) d x\right)^{\frac{1}{2}} .
\end{aligned}
$$

This implies

$$
\begin{aligned}
\left(\int_{\dot{\omega}(-N, N)} B(U, U) d x\right)^{\frac{1}{2}} & \leq\left(\sum_{m=-N}^{N} \int_{\dot{\omega}(m-1, m)} B\left(V^{m}, V^{m}\right) d x\right)^{\frac{1}{2}} \\
& \leq C_{1}\left(\sum_{m=-N}^{N}\left\|e\left(V^{m}\right)\right\|_{L^{2}(\dot{\omega}(m-1, m))}^{2}\right)^{\frac{1}{2}}
\end{aligned}
$$

Applying estimates (5.9) we see that the sum above is bounded by

$$
C_{2}\left(\|f\|_{L^{2}(\dot{\omega}(m-1, m))}^{2}+\sum_{m=-N}^{N}\left\|\psi_{m}\right\|_{L^{2}\left(\tilde{\Gamma}_{m}\right)}^{2}\right)^{\frac{1}{2}} .
$$

Estimating the left-hand side from below by the norm of $e(U)$ we finish the proof

Using the lemma above we prove the following

Theorem 5.2. Let $u$ be a periodic in $\hat{x}$ solution of

$$
L u=f_{0}+\partial_{i} f_{i}
$$

in $\omega\left(t_{1}, t_{2}\right)$, where $t_{2}>t_{1}+2$ for integers $t_{1}<0$ and $t_{2}>0$. Then for any integer $s, h>0$ such that $s-h>t_{1}$ and $s+1+h<t_{1}$ the estimate

$$
\begin{aligned}
\int_{\dot{\omega}(s, s+1)}|e(u)|^{2} d x & \\
\leq & C\left[e^{-A h} \int_{\dot{\omega}(s-h, s+1+h)}|e(u)|^{2} d x+\sum_{m=0}^{2 h+1}+\int_{\dot{\omega}(s-h, s-h+m)}\left(\left|f_{0}\right|^{2}+\left(f_{i}, f_{i}\right)\right) d x\right. \\
+ & \left.f_{0} d x-\left.\int_{\dot{\Gamma} \cdot-h} f_{i} d \hat{x} s\right|^{2}\right]
\end{aligned}
$$


holds with $C$ independent of $s$ and $h$. Here $A$ is the constant from Theorem 1.

Proof . Let $U$ be a periodic in $\hat{x}$ solution to the problem

$$
\begin{aligned}
L U & =f_{0}+\partial_{i} f_{i} & & \text { in } \omega(s-h, s+h+1) \\
\sigma(U) & =\phi+f_{n} & & \text { on } \Gamma_{s-h} \\
\sigma(U) & =\psi-f_{n} & & \text { on } \Gamma_{s+h+1}
\end{aligned}
$$

where $\psi$ and $\phi$ are constant vectors chosen as

$$
\begin{aligned}
\int_{\dot{\Gamma}_{\lrcorner+n+1}} \psi+\int_{\dot{\Gamma}_{,+h+1}} f_{n} d \hat{x} & =P(s+h+1, u) \\
-\int_{\dot{\Gamma}_{,-\Lambda}} \phi+\int_{\dot{\Gamma}_{,-\Lambda}} f_{n} d \hat{x} & =P(s-h, u) .
\end{aligned}
$$

These equations yield

$$
\left.\begin{array}{l}
\psi=P(s+h+1, u)-\int_{\dot{\Gamma}_{,+h+1}} f_{n} d \hat{x} \\
\phi=-P(s-h, u)+\int_{\dot{\Gamma}_{--h}} f_{n} d \bar{x} .
\end{array}\right\}
$$

With this choice of $\phi$ and $\psi$ the solvability condition from Lemma 5.1 is satisfied, so there exists a unique $U$ satisfying the a priori estimate

$$
\begin{aligned}
& \|e(U)\|_{L^{2}(\dot{\omega}(s-h, s+h+1))}^{2} \\
& \quad \leq C\left[\int_{\dot{\omega}(s-h, s+h+1)}\left(\left|f_{0}\right|^{2}+\left(f_{i}, f_{i}\right)\right) d x+\sum_{m=0}^{2 h+1} \int_{\hat{\Gamma}_{,-h+m}}\left|\psi_{m}\right|^{2} d \hat{x}\right]
\end{aligned}
$$

with $C$ independent of $s$ and $h$. The functions $\psi_{m}$ are defined by

$$
\psi_{m}=\int_{\dot{\omega}(s-h, s+h+1)} f_{0} d x-\int_{\hat{\Gamma}_{,-h+m}} \psi d \hat{x},
$$

where $m=0,1, \ldots, 2 h+1$. Using (5.11) together with the formula

$$
P(s+h+1, u)=P(s-h, u)+\int_{\dot{\omega}(s-h, s+h+1)} f_{0} d x+\int_{\hat{\Gamma}_{,+h+1}} f_{n} d \hat{x}-\int_{\hat{\Gamma}_{,-h}} f_{n} d \hat{x}
$$

we get

$$
\psi_{m}=-\int_{\dot{\omega}(s-h, s+h+1)} f_{0} d x-P(s-h, u)-\int_{\hat{\Gamma}_{,-h}} f_{n} d \hat{x}
$$

Moreover, $u-U$ is a solution of the viscoelasticity system satisfying conditions of Theorem 5.1. Hence,

$$
\int_{\dot{\omega}(s, s+1)}|B(u-U, u-U)| d x \leq e^{-A h} \int_{\hat{\omega}(s-h, s+h+1)}|B(u-U, u-U)| d x .
$$

Estimating $B(u-U, u-U)$ from below by $c|e(u-U)|^{2}$ and then using the triangle inequality we get

$$
\int_{\dot{\omega}(s, s+1)}|e(u)|^{2} d x \leq C\left[e^{-A h} \int_{\dot{\omega}(s-h, s+h+1)}|e(u)|^{2} d x+\int_{\dot{\dot{\omega}(s-h, s+h+1)}}|e(U)|^{2} d x\right] .
$$

Application of the apriori estimate for $e(U)$ from Lemma 5.1 completes the proof 
As a consequence, we derive

Theorem 5.3. Let $f_{i}$ be vector function satisfying the inequality

$$
\sum_{i=0}^{n}\left\|f_{i}\right\|_{L^{2}(\dot{\omega}(s, s+1))}+\left\|f_{n}\right\|_{L^{2}\left(\hat{\Gamma}_{0}\right)} \leq c e^{-a s} \quad(s \in \mathrm{N})
$$

where $c$ and $a$ are positive constants independent of $s$. Let $u$ be a periodic solution of the system $L u=f_{0}+\partial_{i} f_{i}$ in $\omega(0, \infty)$ such that $P(0, u)=-\int_{\dot{\omega}(0, \infty)} f_{0} d x+\int_{\Gamma_{0}} f_{n}$,

$$
\|e(u)\|_{L^{2}\left(\dot{\omega}\left(0, \frac{3,+1}{2}\right)\right)} \leq c e^{\frac{(\Lambda-\delta) \mathcal{L}}{2}} \quad(s \in \mathbf{N})
$$

where $c$ is a constant independent of $s, A$ is the constant from Theorem 5.1 , and $\delta$ is a constant such that $0<\delta \leq A$. Then there exist constants $C_{1}, C_{2}$ and $a_{1}, a_{2}$ independent of $s$ and a constant vector $w$ such that

$$
\begin{aligned}
\|e(u)\|_{L^{2}(\dot{\omega}(s, s+1))} & \leq C_{1} e^{-a_{1} s} \\
\|u-w\|_{L^{2}(\dot{\omega}(s, s+1))} & \leq C_{2} e^{-a_{2} s} .
\end{aligned}
$$

Proof. Integrating by parts, we have

$$
\begin{aligned}
P(s, u) & =P(0, u)+\int_{\dot{\omega}(0, s)} f_{0} d x-\int_{\hat{\Gamma}_{0}} f_{n} d \hat{x}+\int_{\hat{\Gamma}_{\bullet}} f_{n} d \hat{x} \\
& =-\int_{\dot{\omega}(s, \infty)} f d x+\int_{\hat{\Gamma}_{,}} f_{n} d \hat{x} .
\end{aligned}
$$

Hence, by virtue of (5.12), $|P(s, u)| \leq c e^{-b s}$. Using the apriori estimate from Theorem $5.2,(5.12)$ and (5.13), we obtain (5.14).

Let $\chi S$ denote a characteristic function fo the set $S$. Consider a periodic in $\hat{x}$ solution of the system

$$
\left.\begin{array}{rlrl}
L v & =\chi_{\dot{\omega}(s, s+1)}-\chi_{\dot{\omega}(s+1, s+2)} & & \text { in } \omega(s, s+2) \\
\sigma(v) & =0 & & \text { on } \partial \omega(s, s+2) .
\end{array}\right\}
$$

Also, set

$$
w_{s}=\int_{\dot{\omega}(s, s+1)} u d x
$$

Choosing $v$ as test function in the basic integral identity and using the Schwarz inequality, we have

$$
\begin{aligned}
\left|w_{s}-w_{s+1}\right| & =\left|\int_{\dot{\omega}(s, s+2)} B(u, v) d x\right| \\
& \leq\left(\int_{\dot{\omega}(s, s+2)}|B(v, v)| d x\right)^{\frac{1}{2}}\left(\int_{\dot{\omega}(s, s+2)}|B(u, u)| d x\right)^{\frac{1}{2}}
\end{aligned}
$$


The application of the second Korn inequality yields $\|B(v, v)\|_{L^{2}(\dot{w}(s, s+2))} \leq C$ where $C$ is independent of $s$. Then

$$
\left|w_{s}-w_{s+1}\right| \leq c e^{-a_{0} s}
$$

where $a_{0}$ is positive and independent of $s$. This implies the existence of $w_{\infty}=\lim _{s \rightarrow \infty}$. Also, we have

$$
\left|w_{9}-w_{9+t}\right| \leq k e^{-a_{0} s}
$$

where $k, a_{0}$ are independent of $s, t$. Therefore, we can let $t \rightarrow \infty$ and obtain

$$
\left|w_{s}-w_{\infty}\right| \leq k e^{-a_{0} s}
$$

Now

$$
\begin{aligned}
\left\|u-w_{\infty}\right\|_{L^{2}(\dot{\omega}(s, s+1))} & \leq\left\|u-w_{s}\right\|_{L^{2}(\dot{\omega}(s, s+1))}+\left\|w_{s}-w_{\infty}\right\|_{L^{2}(\dot{\omega}(s, s+1))} \\
& \leq k_{1}\left[\|e(u)\|_{L^{2}(\dot{\omega}(s, s+1))}+e^{-a_{0} s}\right]
\end{aligned}
$$

where $k_{1}$ is independent of $s$. Now application of (5.14) yields (5.15)

The next result is an existence theorem of the type needed for the construction of the boundary layer. Consider the problem

$$
L u=f_{0}+\partial_{i} f_{i}
$$

in $\omega(-\infty, \infty)$. We assume that $f_{i} \in L^{2}\left(\hat{\omega}\left(t_{2}, t_{1}\right)\right.$ for all $t_{1}<0$ and $t_{2}>0$, and periodic in $\hat{x}$.

Theorem 5.4. Suppose

$$
\sum_{i=0}^{n}\left\|f_{i}\right\|_{L^{2}(\dot{\omega}(-s, s))}^{2}+\left\|f_{n}\right\|_{L^{2}\left(\dot{\Gamma}_{\bullet}\right)}^{2}+\left\|f_{n}\right\|_{L^{2}\left(\dot{\Gamma}_{\bullet}\right)}^{2} \leq M e^{(A-\delta)|s|} \quad(s \in \mathbf{N})
$$

where $M$ and $\delta$ are constants from Theorem, $0<\delta \leq A$. Then for any constant vector $q$ there exists a solution of problem (5.17) such that $P(0, u)=q$ and the estimate

$$
\|e(u)\|_{L^{2}(\dot{\omega}(-k, k))}^{2} \leq C\left(M e^{\left(A-\delta_{1}\right)|k|}+\left|k \| q-\int_{\dot{\Gamma}_{0}} f_{n} d \hat{x}\right|\right)^{2} \quad(k \in \mathrm{N})
$$

holds where $C$ is independent of $k$ and $\delta_{1} \in(0, \delta)$.

Proof. Let $v^{N}$ be a solution to the Neumann problem (5.3) with

$$
\begin{aligned}
& \psi=q+\int_{\dot{\omega}(0, N)} f_{0} d x-\int_{\dot{\Gamma}_{0}} f_{n} d \hat{x} \\
& \phi=-q+\int_{\dot{\omega}(0,-N)} f_{0} d x+\int_{\dot{\Gamma}_{0}} f_{n} d \hat{x} .
\end{aligned}
$$


The vectors $\psi_{m}$ from Lemma 5.1 are determined by

$$
\begin{aligned}
\psi_{-N} & =\phi \\
\psi_{m} & =\int_{\dot{\omega}(m, N)} f_{0} d x-\int_{\hat{\Gamma}_{N}} \psi d \hat{x}=-q+\int_{\dot{\omega}(m, 0)} f_{0} d x+\int_{\hat{\Gamma}_{0}} f_{n} d \hat{x} \\
\psi_{N} & =-\psi
\end{aligned}
$$

where $m=-N+1, \ldots, n-1$. By Lemma 5.1 , there exists a unique $v^{N}$ satisfying the estimate

$$
\|e(u)\|_{L^{2}(\dot{\omega}(-N, N))}^{2} \leq c\left[\sum_{i=0}^{n}\left\|f_{i}\right\|_{L_{\dot{\omega}(-N, N)}^{2}}+\sum_{m=-N}^{N}\left|q-\int_{\dot{\Gamma}_{0}} f_{n} d \hat{x}-\int_{\dot{\omega}(m, 0)} f_{0} d x\right|^{2}\right] .
$$

The function $v^{N+k+1}-v^{N+k}$ satisfies the conditions of Theorem 5.1. Hence, we have an estimate

$$
\begin{aligned}
& \left\|e\left(v^{N+k+1}-v^{N+k}\right)\right\|_{L^{2}(\dot{\omega}(-k, k))}^{2} \\
& \leq C e^{-A N}\left[\sum_{i=0}^{n}\left\|f_{i}\right\|_{L_{\dot{\omega}(-N-k-1, N+k+1)}^{2}}\right. \\
& \left.\quad+\sum_{m=-N-k-1}^{N+k+1}\left|q-\int_{\Gamma_{0}} f_{n} d \hat{x}-\int_{\dot{U}(m, 0)} f_{0} d x\right|^{2}\right] .
\end{aligned}
$$

Let us estimate the last sum separately:

$$
\begin{aligned}
& \sum_{m=-N-k-1}^{N+k+1}\left|q-\int_{\dot{\Gamma}_{0}} f_{n} d \hat{x}-\int_{\dot{\omega}(m, 0)} f_{0} d x\right|^{2} \\
& \quad \leq 2(N+k+1)\left|q-\int_{\dot{\Gamma}_{0}} f_{n} d \hat{x}\right|^{2}+2 \sum_{m=-N-k-1}^{N+k+1}|\hat{\omega}(0, m)| \int_{\dot{\omega}(0, m)}\left|f_{0}\right|^{2} d x \\
& \leq 2(N+k+1)\left|q-\int_{\dot{\Gamma}_{0}} f_{n} d \hat{x}\right|^{2}+2 \sum_{m=-N-k-1}^{N+k+1} c m M e^{(A-\delta)|m|} \\
& \leq 2(N+k+1)\left|q-\int_{\dot{\Gamma}_{0}} f_{n} d \hat{x}\right|^{2}+2 C M e^{\left(A-\delta_{1}\right)(N+k+1)}
\end{aligned}
$$

The constant $\delta_{1}$ above is any number from the interval $(0, \delta)$. To obtain the last inequality, we used the estimates for $f_{i}$ from the statement of the theorem, and the fact that $|\hat{\omega}(0, m)| \leq \mathrm{cm}$ where $c$ depends only on the dimension of the space. Then substitution into (5.20) yields

$$
\begin{aligned}
& \left\|c\left(v^{N+k+1}-v^{N+k}\right)\right\|_{L^{2}(\dot{\omega}(-k, k))}^{2} \\
& \quad \leq M_{2}\left[M e^{A k} e^{-\delta_{1}(N+k)}+e^{-A N}(k+N+1)\left|q-\int_{\dot{\Gamma}_{0}} f^{n} d \hat{x}\right|^{2}\right] .
\end{aligned}
$$


The constant $M_{2}$ is independent of $N$ and $k$.

Next, we use this inequality to show that $e\left(v^{k+s}\right)$ for any fixed $k$ is a Cauchy sequence in $L^{2}(\hat{\omega}(-k, k))$. For any $t \geq 0$ and any $s \geq 0$ consider

$$
\begin{aligned}
& \left\|e\left(v^{k+s}-v^{k+s+t}\right)\right\|_{L^{2}(\dot{\omega}(-k, k))}^{2} \\
& \quad \leq \sum_{l=0}^{t-1}\left\|e\left(v^{k+s+l}-v^{k+s+l+1}\right)\right\|_{L^{2}(\dot{\omega}(-k, k))}^{2} \\
& \quad \leq \sum_{l=0}^{t-1}\left[M_{2} M e^{A k} e^{-\delta_{1}(s+l+k)}+e^{-A(s+l)}(k+s+l+1)\left|q-\int_{\dot{\Gamma}_{0}} f^{n} d \hat{x}\right|^{2}\right]^{\frac{1}{2}} \\
& \leq\left(M_{2} M\right)^{\frac{1}{2}} e^{\left(A-\delta_{1}\right) \frac{k}{2}} e^{-\delta_{1} \frac{l}{2}} \sum_{l=0}^{\infty} e^{-\delta_{1} \frac{l}{2}}+M_{3} k^{\frac{1}{2}}\left|q-\int_{\hat{\Gamma}_{0}} f^{n} d \hat{x}\right| \sum_{l=0}^{\infty} e^{-\frac{\hat{A}-\delta_{1}}{2(\cdot+l)}}
\end{aligned}
$$

This shows that the sequence in question is of Cauchy type.

Next we note that all $v^{k+9}$ for $k$ fixed are solutions to the Neumann problems. Hence, they are orthogonal to all rigid displacements. For such functions, the second Korn inequality can be written in the form (see, for instance, [12: Theorem 2.9])

$$
\left\|v^{k+s}\right\|_{H^{\prime}(\hat{\omega}(-k, k))} \leq C_{k} \| e\left(v^{k+s)} \|_{L^{2}(\hat{\omega}(-k, k))} .\right.
$$

Together, the last two inequalities imply the existence of a function $u$ such that $v^{s} \rightarrow u$ in $H^{1}(\hat{\omega}(-k, k))$ for any fixed $k$ as $s \rightarrow \infty$. Substituting $v^{s}$ into the integral identity and taking the limit as $s \rightarrow \infty$ we obtain that $u$ is a solution to the original problem. Setting $s=0$ in (5.22) and taking the limit as $t \rightarrow \infty$ we obtain estimate (5.19). Finally, integrate $\sigma\left(u-v^{s}\right)$ over a thin slab $\omega\left(-\frac{1}{t}, \frac{1}{t}\right)$ including the plane $x_{n}=0$. Then we use the second Korn inequality to estimate this integral in terms of $e\left(u-v^{s}\right)$. Next, divide both sides by the measure of the slab wish equals to $c \frac{1}{t}$ with $c$ depending only on the dimension of the space. Finally, applying diagonal argument and the Lebesgue differentiation theorem, we select a subsequence $v^{s t}$ such that

$$
\frac{1}{c t} \int_{\dot{\omega}(-1 / t, 1 / t)} \sigma\left(u-v^{s t}\right) d x \rightarrow 0 \quad \text { as } s, t \rightarrow \infty .
$$

By definition of the momenta, this yields $P(0, u)=q$

\section{References}

[1] Avellaneda, M., Berlyand, L. and J. F. Clouet: Frequency dependent acoustics of composites with interfaces. To appear in SIAM Journ. Appl. Math.

[2] Auriault, J.-L.: Poroelastic Media. In: Homogenization and Porous Media (ed.: U. Hornung). New York: Springer 1997.

[3] Biot, M. A.: Theory of propagation of elastic waves in a fluid-saturated porous solid. Part I: Lower frequency range, and Part II: Higher frequency range. J. Acoust Soc. Am. 28 (1956), $168-178$ and $179-191$. 
[4] Biot, M. A.: Mechanics of deformation and acoustic propagation in porous media. J. Appl. Phys. 33 (1962), $1482-1498$.

[5] Burridge, R. and J. B. Keller: Poroelasticity equations derived from microstructure. J. Ac. Soc. Am. 70 (1981), $1140-1147$.

[6] Gilbert, R., Gnelecoumbaga, S. and G. Panasenko: Wave propagation in a system: porous media with Dirichlet's condition on the boundary-continuous media. In: Proc. Third Int. Congr. Acoustics (ed.: G. Cohen). New York: INRIA and SIAM Publ. 1995, $449-455$.

[7] Gilbert, R. and A. Micelić: Homogenizing acoustic properties of the seabed. Preprint.

[8] Hörmander, L.: Analysis of Linear Partial Differential Operators, Vol. I. New York: Springer 1983.

[9] Jikov, V. V., Kozlov, S. M. and O. A. Oleinik: Homogenization of Differential Operators and Integral Functionals. New York: Springer-Verlag 1994.

[10] Levy, T.: Fluids in porous media and suspensions. In: Homogenization Techniques in Composite Media (Lecture Notes in Physics: Vol. 272). New York: Springer-Verlag 1987, 84-120.

[11] Oleinik, O. A. and G. A. Yosifian: On the asymptotic behavior at infinily of solutions in linear elasticity. Arch. Rat. Mech. Anal. 78 (1982), 29 - 55.

[12] Oleinik, O. A., Shamaev, A. S. and G. A. Yosifian: Mathematical Problems in Elasticity and Homogenization. New York: North-Holland 1992.

[13] Panasenko, G. P.: Higher order asymptotics of solutions of contact problems for periodic structures (in Russian). Matem. Sbornik 101 (1979), 505 - 538.

[14] Sanchez-Palencia, E.: Non-Homogeneous Media and Vibration Theory. Springer Lect. Notes Phys. 129 (1980), 1 - 398.

Received 01.02.1999 